\title{
ASKEDAL, JOHN OLE ET AL. (HG.): NORSK GRAMMATIKK. RIKSMAL OG MODERAT BOKMAL. [REVIDERT UTGAVE AV GORGUS COWARD, RIKSMÅLSGRAMMATIKK], 2. OPPLAG. OSLO: DET NORSKE AKADEMI FOR SPRÅK OG LITTERATUR OG KUNNSKAPSFORLAGET, 2015
}

Der Titel verrät es schon: Norwegisch ist nicht gleich Norwegisch. Während vergleichbare Werke in anderen Sprachen mit Titeln wie „Deutsche Grammatik“ oder „Gramatyka języka polskiego“ auskommen, muss „Norsk grammatikk“ zwangsläufig mit einen Untertitel versehen werden, damit deutlich wird, welche der beiden amtlichen Schriftsprachen (Bokmål oder Nynorsk) gemeint ist; und dass es selbst innerhalb dieser Schriftsprachen noch erheblichen Spielraum gibt, was Rechtschreibung und grammatische Formen anbelangt, zeigt das Attribut „moderat" vor Bokmål nur allzu deutlich. Wenn es ein „moderates“ Bokmål gibt, gibt es folglich auch ein anderes, das in dem hier besprochenen Werk keine Berücksichtigung findet: das sog. ,radikale“ Bokmål - ein unmittelbares Ergebnis der besonders Mitte des 20. Jahrhunderts in Norwegen praktizierten Sprachpolitik, die darauf hinarbeitete, die beiden amtlichen Schriftsprachen - Bokmål und Nynorsk - irgendwann zu EINER norwegischen Schriftsprache (dem sog. „Samnorsk“) zu verschmelzen. Das Fiasko dieser Poli-

* Gero Lietz - dr., ukończył studia skandynawistyczne na Uniwersytecie w Greifswaldzie w 1988 roku. W 1991 roku obronił pracę doktorską z dziedziny skandynawistyki (temat rozprawy: Problem nazw własnych w przekładzie współczesnej literatury norweskiej na język niemiecki). W latach 1996-2001 i 2005-2010 pracował jako lektor DAAD w Instytucie Lingwistyki Stosowanej i w Instytucie Filologii Germańskiej UAM w Poznaniu. Od 2014 zatrudniony jest w Centrum Interdyscyplinarnych Studiów o Polsce na Uniwersytecie Europejskim Viadrina we Frankfurcie nad Odrą. W latach 2014-2016 był st. wykładowcą w Katedrze Skandynawistyki Uniwersytetu Szczecińskiego. Zajmuje się m.in. onomastyką, fonetyką, translatoryką; e-mail: gero_lietz_ffo@gmx.de 
tik zeichnete sich in der zweiten Hälfte des 20. Jahrhunderts immer deutlicher ab, dennoch wurde sie offiziell erst im Jahre 2002 aufgegeben - durch die Streichung entsprechender Passagen im Gesetz über den Norwegischen Sprachrat. Das eigentlich Interessante an der vorliegenden Grammatik ist jedoch, dass die Norwegische Akademie für Sprache und Literatur (seit den 1950er Jahren zuständig für die Normierung der oft als ,privat“ und ,inoffiziell“" bezeichneten Riksmål-Norm¹) hier das moderate Bokmål in seine Normierungs- und Standardisierungsarbeit einbezieht. Das „inoffizielle“ Riksmål mit seiner seit jeher stringenteren und am wirklichen schriftsprachlichen Gebrauch orientierten Norm bietet sich damit zugleich als Orientierung für die künftige standardsprachliche Normierung des offiziellen Bokmål an.

Das Buch geht auf die 1958 erstmals erschienene Kortfattet riksmålsgrammatikk zurück, die - verfasst von dem verdienstvollen Norwegischlehrer, Bildungs- und Sprachpolitiker Gorgus Coward (1912-1994) ${ }^{2}$ - mehrere Neuauflagen erlebte und 1986 in erweiterter Form unter dem Titel Riksmålsgrammatikk. Med en sproghistorisk innledning og en rettskrivningslcere herausgegeben wurde. Diese Ausgabe von 1986 liegt der jetzigen Neubearbeitung zugrunde. Seit den 1980er Jahren hat es einige Bewegung in der norwegischen Sprachpolitik gegeben. Der 2002 beschlossene, oben erwähnte Abschied von der Samnorsk-Politik des norwegischen Staates war spätestens mit der Bokmål-Reform von 1981 eingeleitet worden, bei der viele seit 1938 verbotene Riksmål-Formen wieder Eingang in die offizielle Bokmål-Norm fanden und sich so der Abstand zwischen der offiziellen Norm und der ,inoffiziellen“ Riksmål-Norm erheblich verkleinerte. Die Bokmål-Reform von 2005 führte schließlich $\mathrm{zu}$ einer noch umfassenderen und weitreichenden Rehabilitierung vieler traditioneller Riksmål-Formen (Zulassung einer Reihe von Präteritumsformen bei den Verben, Abschaffung etlicher ,wenig verwendeter“ Formen, Abschaffung der Unterteilung der Bokmål-Norm in sog. Haupt- und Nebenformen mit jeweils bestimmten Anwendungsbereichen), so dass sich heute, nicht zuletzt auch dank einer Reihe von

1 Gegner des Riksmål bezeichnen diese Norm oft abwertend als ,privat“, da sie sich nicht auf politische Beschlüsse und öffentliche Vorschriften stützen könne und als solche keine demokratische Legitimation besitze, vgl. John Ole Askedal, «Opprydning må til i bokmålsnormen», in: Ordet 4, 2015, 5-6 (5). Die Riksmål-Bewegung hat sich stets vehement gegen die Samnorsk-Politik ausgesprochen, vgl. eine Aussage des späteren Vorsitzenden des Riksmålverbandes, des Dichters Arnulf Øverland (1889-1968) der in einer Rede im März 1940 (Titel: „Ist unsere Sprache abgeschafft?“) die sog. Rechtschreibreform von 1938 scharf attackierte: „Das, was Rechtschreibreform von 1938 genannt wird, ist keine Rechtschreibreform, es ist eine Aufhebung unserer zwei, jede für sich vorzüglichen, Schriftsprachen, es ist die Einführung einer ganz neuen Sprache [...]“, zit. nach FinnErik Vinje, Et språk i utvikling (Oslo: Aschehoug, 1978). Befürchtet wurde durch die Samnorsk-Politik eine Schwächung beider norwegischen Schriftsprachen (Riksmål/Bokmål und Nynorsk), die jede für sich einen wertvollen Teil der norwegischen Kultur und Tradition darstellen.

2 Zur Biographie von Gorgus Coward vgl. https://nbl.snl.no/Gorgus_Coward (28.3.2016). 
Beschlüssen der Norwegischen Akademie für Sprache und Literatur, die Unterschiede zwischen dem Riksmål und dem moderaten Bokmål auf „schätzungsweise 150 weniger gebräuchliche Einzelwörter und Wortformen sowie auf eine bestimmte Zahl von Zusammensetzungen und Zusammenschreibungen" (S. 12) reduziert haben. Die Sprache des Buches trägt dieser Entwicklung in den letzten dreißig Jahren Rechnung: Der ursprüngliche Text von Coward ist einer gründlichen Durchsicht unterzogen worden, er befindet sich auf dem Stand der Riksmål-Norm von 2008 und entspricht daher im Wesentlichen dem moderaten Bokmål, denn die heutige Riksmål-Norm stellt im Großen und Ganzen eine Teilmenge der Bokmål-Norm dar. Zum aktuellen Stand der Bemühungen um eine gemeinsame Standardsprache heißt es im Geleitwort der Herausgeber: „Sind im Bokmål unter mehreren zugelassenen Formen auch solche, die laut Riksmål-Norm zulässig sind, werden sich diejenigen, die moderates Bokmål schreiben wollen, in der Regel für die mit dem Riksmål gemeinsame Form entscheiden. In den wenigen Fällen, in denen das nicht möglich ist, müssen sie eine der offiziellen Formen verwenden. Zurzeit wird daran gearbeitet, die Unterschiede zwischen Riksmål und moderatem Bokmål noch weiter zu reduzieren, insbesondere im Hinblick auf die Schreibweise fremder Wörter.“ (S. 12) ${ }^{3}$ Herausgegeben wird das Buch in Zusammenarbeit mit der erwähnten Norwegischen Akademie für Sprache und Literatur, die sich bis 2013 Det Norske Akademi for Sprog og Litteratur schrieb, sich auf dem Umschlag der vorliegenden Grammatik aber bereits mit der neuen Schreibweise Det Norske Akademi for Språk og Litteratur präsentiert. Auch dies ist eine Geste, die zeigt, dass die Riksmål-Bewegung es ernst meint mit ihrem Wirken für die Schaffung einer attraktiven, modernen europäischen Standardsprache. Das moderate Bokmål ist in diesem Streben der natürliche Verbündete.

Die Kapitelstruktur orientiert sich hinsichtlich der grammatischen Beschreibung an der Ausgabe von 1986. Innerhalb der einzelnen Kapitel (Wortarten, Wort- und Satzgliedstellung) haben die Herausgeber Aktualisierungen vorgenommen, was die Reihenfolge der Einzelthemen, Überschriften, die Auswahl der Beispiele und auch die grammatische Terminologie anbelangt. Eingearbeitet in die Darstellung ist auch die seit 2005 für den Gebrauch an norwegischen Schulen vom Bildungsministerium empfohlene neue grammatische Terminologie, allerdings immer mit Hinweisen auf die traditionellen Termini, denen dann im Weiteren der Vorzug bei der gramma-

3 Man muss schon sehr genau suchen, um im Text der vorliegenden Grammatik reine Riksmål-Formen oder Schreibweisen zu finden, die im offiziellen Bokmål nicht zulässig sind. Einige wenige gibt es, z.B. idag (S. 9; Bokmål i dag), femogtyve (S. 57; Bokmål tjuefem), kanhende (S. 131, Bokmål kan hende) u.a. In anderen Fällen schreibt die Riksmål-Norm e i n e Schreibweise vor, während im Bokmål Wahlfreiheit besteht, z.B. hverken (Bokmål hverken oder verken), antagelig (antagelig/ antakelig), lignende (lignende/liknende). 
tischen Beschreibung gegeben wird. Dadurch wird zweifellos die Verständlichkeit der Darstellung erhöht, vgl. etwa die terminologischen Erläuterungen zu den Begriffen hovedsetning, bisetning, leddsetning, periode, helsetning (S. 15 f.) sowie die nachfolgende Verwendung der traditionellen Begriffe hovedsetning (Hauptsatz) und bisetning (Nebensatz) im Kapitel zur Wort- und Satzgliedstellung (S. 153-159). Die gleiche Verfahrensweise verwenden die Herausgeber in Kapitel 3, wo im einleitenden Abschnitt über die verschiedenen Tempora des Verbs zunächst eine Übersicht über die traditionell verwendeten Termini und die neuen Termini gegeben wird (z.B. perfektum, pluskvamperfektum vs. presens perfektum, preteritum perfektum), um dann in der weiteren Beschreibung (z.B. der Verwendung der norwegischen Tempora ab S. 32) ausschließlich die traditionellen Termini zu gebrauchen. Einige Abschnitte wurden für die jetzt vorliegende Überarbeitung von Cowards Grammatik neu geschrieben, so u.a. der sehr lesenswerte Abschnitt 3.7 („Forstavelsesverb vs. partikkelverb“, S. 46-48), in dem in aller Kürze das Wichtigste zu der im Norwegischen sehr komplexen Problematik von Präfixverben und Partikelverben gesagt wird. Einige Kapitel im zweiten Teil des Buches gehen über die direkte grammatische Beschreibung hinaus: „Zeichensetzung“ (S. 160-166), „Wortwahl und Stil“ (S. 167-172), ,Rechtschreibregeln“ (S. 173-190) sowie ein sehr instruktives und lesenswertes Kapitel über die Normierungsgeschichte des Riksmål/Bokmål seit 1814 (dem Jahr der Auflösung der dänisch-norwegischen Union (S. 191-229). Im Anhang findet sich neben einem Sachregister und der Literaturliste auch ein sehr nützliches Verzeichnis grammatischer Termini (S. 230-240). ${ }^{4}$

Immer wieder wird in der grammatischen Beschreibung auf Fragen der Sprachentwicklung hingewiesen, sei es im Zusammenhang mit der heute außer Gebrauch gekommenen Höflichkeitsform der Anrede (8.1.11 Høflig tiltale, S. 96-98) oder mit dem Hinweis auf Wortformen, die für das Verständnis älterer Texte wichtig sind, die jedoch heute nur noch selten oder in archaisierender Funktion verwendet werden, wie z.B. das reziproke Pronomen hinannen (heute: hverandre, S. 103) oder das Pronomen sådan, über das es heißt, dass es sowohl adjektivisch als auch substantivisch verwendet werden kann und dann ,einen stilistischen Wert hat, der über

4 Das Verzeichnis enthält sowohl die traditionelle als auch die neue grammatische Terminologie mit entsprechenden Verweisen. Auf S. 240 ist den Herausgebern ein bedauerlicher Irrtum unterlaufen: Der Begriff utrum (steht für ,gemeinsames Geschlecht', ,Nicht-Neutrum ${ }^{\star}$ ) wird mit intetkjønn (,Neutrum') erklärt. Stehen müsste als Erklärung felleskjønn. Trotz der Berichtigung von Druckfehlern in der 2. Auflage (vgl. Vorwort zur 2. Auflage, S. 13) sind einzelne Fehler offenbar nicht gefunden worden. So fehlt auf S. 61 unten in dem Beispiel „,den epokegjørende oppdagelsen til [...] av våre fremste og internasjonalt mest kjente forskere“ das Wörtchen „en“. Auch auf S. 66 oben hat sich ein Fehler eingeschlichen: Statt „gjør“ muss es „gjøre“ heißen. Solche Flüchtigkeitsfehler gehören allerdings zu den Ausnahmen. 
das neutral Alltägliche hinausgeht" (S. 115). Hingewiesen wird auch auf das Relativpronomen hvilken, das in älteren Texten wesentlich öfter anzutreffen war als heute (S. 110). Ebenfalls veraltet seien die Relativadverbien hvori, hvorpå und hvortil (S. 110), während hvorav noch „höchst lebendig“ sei - und zwar zur Angabe partitiver Verhältnisse: Minst ni mennesker, hvorav to barn, mistet livet i ulykken (S. 111). Zum Verhältnis von ingen und ikke noen wird festgestellt, dass die Platzierung eines Objekts mit ingen vor der infiniten Verbform heute als veraltet oder zumindest als stark abweichend von der neutralen Stilebene gelten muss. Für Sätze wie Hun hadde ingen beskjed fät oder De hadde ingen problemer sett sollte man heute besser formulieren: Hun hadde ikke fått noen beskjed und De hadde ikke sett noen problemer (S. 120 f.).

Im Zentrum der Darstellung steht jedoch die moderne norwegische Gegenwartssprache und ihre Grammatik, die sich für das Riksmål - das liegt in der Natur dieser Norm - wesentlich einfacher und übersichtlicher darstellen lässt als für das Bokmål, zumindest wenn man die vollständige offizielle Bokmål-Norm berücksichtigen wollte - mit ihrer Fülle von Varianten, von denen viele nur sehr selten oder nie verwendet werden, die aber trotzdem - sozusagen als Relikte der Samnorsk-Politik - in den amtlichen Wörterbüchern und Grammatiken zu finden sind. Das betrifft sowohl den Wortschatz ${ }^{5}$ als auch das grammatische System.

Als Beispiel für sprachsystematische Besonderheiten des Riksmål und des moderaten Bokmål gegenüber dem Nynorsk und dem „,radikalen“ Bokmål kann die Frage des Genus der Substantive dienen. Die offizielle Bokmål-Norm (die ja auch die ,radikale"Variante mit einschließt) stellt es dem Sprachbenutzer seit 1981 frei, generell bei allen Substantiven, die in den Dialekten den bestimmten Artikel - $a$ (für Feminina) haben, entweder $-a$ oder auch $-e n$ zu verwenden (dementsprechend darf bei diesen Wörtern auch der unbestimmte Artikel ei statt en verwendet werden). ${ }^{6}$ Die moderne Riksmål-Norm ist hier einerseits weniger liberal, andererseits jedoch viel näher an

5 Askedal (wie Anm. 1) verweist insbesondere auf die „,mechanisch konstruierten“ Möglichkeiten für Komposita im offiziellen Bokmål, wie etwa im Falle der Zusammensetzung skogbunn (,Waldboden'), das lt. offizieller Norm auch skogbotn, skaubunn oder skaubotn heißen darf. Beispiele dieser Art finden sich sehr viele, vgl. das umfassende Wörterbuch Bokmålsordboka (http://www.nob-ordbok.uio.no/), in dem sämtliche offiziell gültigen Formen aufgeführt sind. Sucht man dort z.B. nach dem Wort meningsløs, erhält man in alphabetischer Reihenfolge vier offiziell gleichberechtigte Varianten: meiningslaus, meiningsløs, meningslaus, meningsløs. Die mit Abstand am häufigsten gebrauchte Form meningsløs (wenn man mal von der eigentlich reinen Nynorsk-Form meiningslaus absieht) erscheint an letzter Stelle. Kommentieren lässt sich das am besten mit dem Lexem selbst, das im Deutschen so viel wie, sinnlos' bedeutet.

6 Seit der Rechtschreibreform von 1938 hatten in der offiziellen Bokmål-Norm Hunderte obligatorische $a$-Wörter existiert. 1981 wurde dann der bestimmte Artikel -en für diese Substantive wieder zugelassen, was weithin als eine Art „Rehabilitierung“ von Riksmål-Formen angesehen wurde. 
der schriftsprachlichen Realität. Riksmål und moderates Bokmål gehen (wie auch das Dänische und Schwedische) von einem System mit nur zwei Genera aus: Utrum (,felleskjønn' $=$,Nicht-Neutrum') und Neutrum. Der weibliche unbestimmte Artikel $e i$ ist somit in der Riksmål-Norm gar nicht vorgesehen. Das entspricht einer verbreiteten schriftsprachlichen Praxis und macht das Genussystem nicht zuletzt auch für ausländische Lerner des Norwegischen übersichtlicher. Zugleich zeigt die heutige Norm des Riksmål, dass sie eine realitätsnahe, moderne Norm ist. Im Unterscheid nämlich zur schematischen Gleichsetzung der bestimmten Artikel -en und $-a$ bei „eigentlichen Feminina“ bzw. „Kryptofeminina“ (S. 50) in der offiziellen Bokmål-Norm (im heutigen Bokmål gibt es also offiziell kein einziges obligatorisches $a$-Wort mehr!) umfasst die Riksmål-Norm eine Liste von ca. 50-60 Substantiven, die obligatorisch mit dem bestimmten Artikel $-a$ verwendet werden, z.B. $k u(k u a)$, øy (øya), bikkje (bikkja). Darüber hinaus gibt es in der Riksmål-Norm eine Liste von ca. 200 Substantiven, die entweder $-a$ oder $-e n$ als bestimmten Artikel erhalten können. Im Rahmen des Zwei-Genera-Systems des Riksmål und des moderaten Bokmål wird die Verwendung des ursprünglich weiblichen Artikels als „eigene Flexionsklasse innerhalb des Utrum“ (S. 50) klassifiziert. Viele Bokmål-Nutzer (die Riksmål-Nutzer sowieso) schreiben also z.B. ,en $k u^{\prime \prime}$ oder ,en $\varnothing y^{\prime \prime}$ (statt „ei“), aber benutzen gleichzeitig kua und øya, was in beiden Normen legitim ist und dem allgemeinen schriftsprachlichen Usus entspricht. Zugleich ist die Riksmål-Norm in diesem Punkt elastisch und pragmatisch. Es wird dem Sprachbenutzer in Abhängigkeit von Stil und Thema des betreffenden Textes anheimgestellt, sogar bei „Kryptofeminina“, die in der Riksmål-Norm ausschließlich als en-Wörter verzeichnet sind, gegebenenfalls den bestimmten Artikel $-a$ zu verwenden.

Eine ebenso formalistische Lösung wie für den Bereich des bestimmten Artikels (generelle Gleichstellung der Artikel $-a$ und $-e n$ für potentielle Feminina) gilt im offiziellen Bokmål für die Endungen des Präteritums und des Partizips Perfekt der Gruppe 1 der schwachen Verben (der sog. kaste-Gruppe): Hier wird es den Sprachbenutzern generell - und zwar ohne stilistische Einschränkungen - freigestellt, entweder die Endung - et oder die in vielen Dialekten verbreitete Endung - $a$ zu gebrauchen (also kastet oder kasta, klatret oder klatra usw.). Das Riksmål zieht in dieser Frage in Übereinstimmung mit dem moderaten Bokmål eine deutliche Grenze: Die Endung $-a$ wird für den schriftsprachlichen Gebrauch dieser Verben prinzipiell ausgeschlossen (S. 24).

Der Grammatik ist anzumerken, dass ihr ursprünglicher Autor Gorgus Coward Pädagoge war. Bewundernswert ist die präzise und zugleich plastische Darstellung bestimmter schwieriger Themen, etwa wenn es um die Unterscheidung von $a$ und og geht (S. 37 f.) oder um die Frage der einfachen und doppelten Bestimmung von Substantiven, wo mit einfachen, einprägsamen Beispielen bestimmte Bedeutung- 
sunterschiede erklärt werden, so z.B. zwischen den ukjente soldat und den ukjente soldaten. Eindrucksvoll wird gezeigt, dass sowohl die einfache Bestimmung als auch die doppelte Bestimmung norwegischer Substantive ein stilistisches Potential besitzen, das nicht ohne Not verschenkt werden sollte. Es ist nicht zuletzt ein sprachpflegerischer Aspekt, der sich geltend macht, wenn festgestellt wird: „Es ist nicht so, dass die Sprache ohne weiteres durch massiven und unkritischen Gebrauch der doppelten Bestimmung mündlicher oder ,natürlicher' oder dass sie durch vermehrten Gebrauch der einfachen Bestimmung ,literarischer" und ,gediegener" würde.“ (S. 69). Zur Sprachpflege gehört auch das Bemühen, durch die Aufrechterhaltung bestimmter Schreibweisen und Endungen weiterhin in der Lage zu sein, Bedeutungsnuancen auszudrücken, zum Beispiel bei der Unterscheidung attributiv gebrauchter Adjektive/ Partizipien (S. 73 f.). Als sehr einleuchtendes Beispiel wird flekket angeführt. Flekket kann zum einen ein von dem Substantiv flekk abgeleitetes Adjektiv sein (auch in der Schreibung flekkete: ,gefleckt, fleckig, mit Flecken'). Zum anderen kann flekket ein von dem Verb flekke (,fletschen') abgeleitetes Partizip sein. Zur Bedeutungsunterscheidung schreibt die Riksmål-Norm im letzteren Falle grundsätzlich die Schreibung -ede (bestimmte Form und Plural) vor, so dass ,gefletschte Zähne' flekkede tenner heißen - im Unterschied zu ,gefleckten Zähnen' in der Schreibung flekkete tenner. Im Bokmål kann diese Unterscheidung in identischer Weise vorgenommen werden, man darf aber laut Bokmål-Norm auch beide Fälle mit der Schreibung flekkete wiedergeben und vergibt sich so die Chance auf Bedeutungsunterscheidung.

Bewusste Sprachpflege ist eines der Hauptanliegen der Verfasser und Herausgeber des Buches und kommt auch im Kontext anderer grammatischer Themen immer wieder zum Vorschein. Genannt seien als Beispiele die Verwendung der Objektform des Personalpronomens han (ham oder han?, S. 92 f.) oder die ,alte“ und „neue“ Zählweise (enogtyve vs. tjueen, S. 84). Interessant ist auch der Kommentar zur Umschreibung des Genitivs mit sin (sog. ,garpegenitiv“, z.B. Petter sin sykkel statt Petters sykkel). Diese stamme aus der Hansezeit und habe trotz ihres „zweifelhaften, unnationalen Ursprungs“" (aus dem Niederdeutschen) Eingang in das offizielle Nynorsk gefunden. $\mathrm{Zu}$ einem natürlichen Teil der Norm des Riksmål und des moderaten Bokmål sei diese Umschreibung jedoch nie geworden, und es gebe ja auch allgemein akzeptierte Alternativen dazu (S. 62).

Zielgruppe dieser Grammatik ist in erster Linie die norwegische Sprachgemeinschaft, der in Gestalt des Riksmål und des moderaten Bokmål eine stringente Norm angeboten wird, die sich wohltuend abhebt von der offiziellen, auch die radikalen Formen beinhaltenden Bokmål-Norm. Größtes Problem der gegenwärtigen Bokmål-Norm ist John Ole Askedal zufolge die große „Menge konstruierter und wenig geläufiger Wortformen und Flexionsmöglichkeiten“, die in der schriftsprachlic- 
hen Realität „nur am Rande oder niemals“ in Erscheinung treten. ${ }^{7}$ Es liegt auf der Hand, dass dieses Problem im Bereich des Norwegischen als Fremdsprache (z.B. an ausländischen Universitäten) oder des Norwegischen als Zweitsprache noch viel relevanter ist. Ausländische Lerner des Norwegischen verfügen im Unterschied zu norwegischen Muttersprachlern über keinerlei dialektale Grundlage, so dass es für sie noch viel weniger einzusehen ist, warum sie im Wörterbuch zuweilen zwischen drei oder vier Rechtschreib- oder Flexionsvarianten wählen sollen, von denen manche bloße Konstrukte oder aber sprachideologische Reste aus der Zeit der Samnorsk-Politik darstellen. Mit ihrer Standardisierungsarbeit leistet die Norwegische Akademie für Sprache und Literatur somit nicht nur eine ungemein wichtige Arbeit für die norwegischen Muttersprachler und Muttersprachlerinnen, sondern auch für die Attraktivität des Norwegischen als Fremdsprache/Zweitsprache. Ausdruck dessen war das Titelbild der ersten Auflage (2013) der hier besprochenen Grammatik, das von einem Rezensenten in der Tageszeitung „Dagbladet“ wie folgt kommentiert wurde: „Den Umschlag des Buches ziert ein Foto gutgelaunter Studierender während des Unterrichts. Die meisten von ihnen haben eine dunkle Haut- und Haarfarbe und lächeln erwartungsvoll in Richtung Bildrand. Vielleicht äußern sie ja auf diese Art und Weise ihre Erleichterung darüber, mit dieser Grammatik endlich eine konzise, gründliche Übersicht zu der Sprache zu haben, der sie in ihrer neuen Kultur faktisch begegnen, obgleich das Buch ja gar nicht direkt an sie gerichtet ist.“ Weiter heißt es: „Menschen mit einer anderen Muttersprache als Norwegisch haben Schwierigkeiten mit all unseren Varianten. Für sie ist eine genug [...]." ${ }^{\text { }}$ Dem ist zuzustimmen, ${ }^{9}$ und zugleich ist zu bedauern, dass das Titelbild der zweiten Auflage nicht mehr diesen internationalen Aspekt in den Vordergrund rückt. ${ }^{10}$ Standardsprachliche Normierung ist aber nicht nur für die betreffende Sprachgemeinschaft selbst, sondern auch für die ausländischen Lerner dieser Sprache von immenser Bedeutung. Das gilt für das Norwegische ganz besonders, denn beherrscht man Norwegisch (insbesondere Riksmål/Bokmål), hat man nicht zuletzt auch in Bezug auf die interskandinavische

7 Askedal (Anm. 1), S. 6. Vgl. Beispiele oben in Anmerkung 5.

8 Jon Rognlien: Undergrunnsgrammatikk. Dagbladet, 11.01.2014, http://www.dagbladet.no/2014/ 01/11/kultur/meninger/kommentar/sprak/ordbok/31226782/ (22.05.2016).

9 Was keine Herabstufung der zweiten amtlichen Schriftsprachen (Nynorsk) bedeuten soll. Norwegisch als Fremdsprache - zumal im Ausland - heißt jedoch in aller Regel Bokmål. Mit „all unseren Varianten" sind wohl auch in erster Linie die Varianten innerhalb der offiziellen Normen des Bokmål und des Nynorsk gemeint, die nicht dazu geeignet sind, den Lernprozess bei ausländischen Lernern zu beschleunigen.

10 Der Umschlag der zweiten Auflage zeigt die Fohlen-Skulptur des Bildhauers Per Hurum, wie sie alljährlich an die Preisträger des Thorleif-Dahl-Preises der Norwegischen Akademie für Sprache und Literatur überreicht wird. 
Kommunikation (im Verhältnis zum Dänischen und Schwedischen) sehr deutliche Vorteile. Spätestens mit der Ausräumung der letzten noch verbliebenen Differenzen zwischen der Riksmål-Norm und dem moderaten Bokmål sollte somit auch daran gedacht werden, die jetzt vorliegende Grammatik noch stärker für die Zwecke des Unterrichts in Norwegisch als Fremdsprache/Zweitsprache aufzubereiten.

Schon jetzt kann ausländischen Norwegisch-Studierenden neben der hier besprochenen Norsk Grammatikk auch das in laufend aktualisierter Fassung erscheinende Wörterverzeichnis Riksmålsordlisten (herausgegeben vom Riksmålsforbundet) sowie vor allem das hervorragende einsprachige Wörterbuch Norsk Ordbok med 1000 illustrasjoner von Tor Guttu ${ }^{11}$ wärmstens empfohlen werden.

11 Riksmålsordlisten, utgitt av Riksmålsforbundet, 8. utgave, Oslo: Kunnskapsforlaget 2015; Guttu, Tor (red.): Norsk Ordbok med 1000 illustrasjoner. Riksmål og moderat bokmål, 2. utgave, Oslo: Kunnskapsforlaget 2010. 
